\title{
Drift instability and tunneling of lattice solitons
}

\author{
Y. Sivan, ${ }^{1}$ G. Fibich, ${ }^{2}$ and B. $\operatorname{Ilan}^{3}$ \\ ${ }^{1}$ Department of Physics and Astronomy, Tel Aviv University, Israel \\ ${ }^{2}$ Department of Applied Mathematics, Tel Aviv University, Israel \\ ${ }^{3}$ School of Natural Sciences, University of California, Merced, California, USA
}

\begin{abstract}
We derive an analytic formula for the lateral dynamics of solitons in a general inhomogeneous nonlinear media, and show that it can be valid over tens of diffraction lengths. In particular, we show that solitons centered at a lattice maximum can be "mathematically unstable" but "physically stable". We also derive an analytic upper bound for the critical velocity for tunneling, which is valid even when the standard Peierls-Nabarro potential approach fails.
\end{abstract}

PACS numbers: $42.65 \mathrm{Jx}, 42.65 \mathrm{Tg}, 03.75 \mathrm{Lm}$

Solitons have been thoroughly studied in diverse fields of physics such as nonlinear optics, BEC, plasma and water waves. By now, the stability and dynamics of solitons in homogeneous media are well understood. The possibility to manufacture transparent materials with spatially varying, high contrast dielectric properties raises new questions regarding stability and dynamics of solitons in inhomogeneous media. In particular, while in a homogeneous medium the solitons can freely move sideways, in an inhomogeneous medium the loss of translation invariance affects the lateral movement of solitons. The problem of lateral movement of lattice solitons is interesting theoretically and important for applications such as all optical switching and quantum information science. It was studied analytically, numerically and experimentally for various media and lattices, see e.g., [1, 2, , 3, 4, [5, [5] However, each of these studies considered a specific nonlinearity, lattice type and dimension.

In this Letter, we provide, apparently for the first time, a unified theory for the mobility of lattice solitons which is valid for any nonlinearity, lattice type and dimension. We show that soliton mobility is intrinsically related to soliton stability, two key properties that so far were studied separately. This relation enables us to compute analytically the rate of drift of solitons initially centered near a lattice maximum, and the restoring force that the lattice exerts upon solitons initially centered near a lattice minimum. In the latter case, our approach provides an upper bound for the critical velocity for tunneling, which is valid even when the standard Peierls-Nabarro potential approach cannot be applied.

All solitons centered at a lattice maximum are "mathematically unstable", as they drift towards the nearest lattice minimum [4, [5, 6, , 7]. However, the ability to compute the magnitude of the drift rate allows us to identify cases in which the drift rate is so small so that the soliton is "physically stable", i.e., the drift instability does not develop over the propagation distance of the experiment. This observation explains why in some experiments solitons centered at a lattice maximum were observed to be stable [3].

Consider the dimensionless nonlinear Schrödinger equation (NLS) with a linear lattice

$$
i A_{z}(z, \mathbf{x})=-\nabla^{2} A-F\left(|A|^{2}\right) A+V(N \mathbf{x}) A,
$$

where $\mathbf{x}=\left(x_{1}, \ldots, x_{d}\right)$ and $V$ is a linear lattice/potential with a characteristic length scale or period $1 / N$. This equation describes propagation in a media with Kerr nonlinearity as well as quintic, cubic-quintic, saturated/photorefractive nonlinearities etc. The variable $z$ denotes the propagation coordinate in nonlinear optics and time coordinate in BEC. Eq. (11) has soliton solutions $A=e^{i \mu z} u(\mathbf{x})$, where $u$ is the solution of

$$
\nabla^{2} u(\mathbf{x})+F\left(|u|^{2}\right) u-V(N \mathbf{x}) u-\mu u=0 .
$$

It is well known that a necessary condition for stability is the slope $(\mathrm{VK})$ condition $\frac{d \mathcal{P}}{d \mu}>0$ where $\mathcal{P}=\int u^{2} d \mathbf{x}$ is the soliton power. Violation of the slope condition leads to a width instability, i.e., small perturbations can lead to large changes of the soliton width, which in some cases result in collapse [4, 5, 6].

If the soliton is centered at a lattice maximum, it can become unstable even if the slope condition is satisfied [3, 4, 5, 6, 7]. Indeed, there is a second condition for stability, the spectral condition, which states that for $A=u e^{i \mu z}$ to be stable, the number of negative eigenvalues of the operator $L_{+, \mu}^{(V)}=-\nabla^{2}+\mu+$ $V(\mathbf{x})-F\left(u^{2}\right)-2 u^{2} F^{\prime}$ should be at most one more than the number of negative eigenvalues of the operator $L_{-, \mu}^{(V)}=-\nabla^{2}+\mu+V(\mathbf{x})-F\left(u^{2}\right)[\underline{8}]$.

A simpler version of the spectral condition was derived in [4] for the case $u>0$. Recall that in a homogeneous medium, $L_{+, \mu}^{(V \equiv 0)}$ has $d$ zero eigenvalues $\lambda_{0, j}^{(V \equiv 0)}=0$ with corresponding eigenfunctions $f_{j}^{(V \equiv 0)}=\frac{\partial Q}{\partial x_{j}}$, where $Q=$ $u^{(V \equiv 0)}$ is the solution of (2) with $V \equiv 0$. The potential $V$ breaks the translation symmetry of the medium. As a result, $\lambda_{0, j}^{(V)}$ can split into $d$ different values. The spectral condition is violated if and only if at least one $\lambda_{0, j}^{(V)}$ attains a negative value [4].

The spectral condition can be derived from the following linear stability analysis [8]. Let $A=$ $e^{i \mu z}(u(\mathbf{x})+h(z, \mathbf{x}))$ where $h(z, \mathbf{x})$ is a small perturbation. Since the instability due to violation of the spectral 
condition originates only from the eigenfunctions $f_{j}^{(V)}(\mathbf{x})$ of $L_{+, \nu}^{(V)}$ which correspond to negative $\lambda_{0, j}^{(V)}$, we can rewrite the perturbation $h$ as

$h(z, \mathbf{x})=c_{1} e^{\Omega_{j} z}\left(f_{j}^{(V)}+i g_{j}^{(V)}\right)+c_{-1} e^{-\Omega_{j} z}\left(f_{j}^{(V)}-i g_{j}^{(V)}\right)$,

where $c_{ \pm 1}$ are constants and $L_{+, \mu}^{(V)} f_{j}^{(V)}=$ $-\Omega_{j} g_{j}^{(V)}, \quad L_{-, \mu}^{(V)} g_{j}^{(V)}=\Omega_{j} f_{j}^{(V)}$. Since $L_{+, \mu}^{(V)} f_{j}^{(V)}=$ $\lambda_{0, j}^{(V)} f_{j}^{(V)}$ and $L_{-, \mu}^{(V)}$ is positive definite,

$$
\Omega_{j}^{2}=-C_{V} \lambda_{0, j}^{(V)}, \quad C_{V}=\frac{\left\langle f_{j}^{(V)}, f_{j}^{(V)}\right\rangle}{\left\langle L_{-, \mu}^{(V)^{-1}} f_{j}^{(V)}, f_{j}^{(V)}\right\rangle}>0 .
$$

Therefore, $\Omega_{j}$ is real (i.e., instability) when $\lambda_{0, j}^{(V)}<0$ and imaginary (i.e., stability) when $\lambda_{0, j}^{(V)}>0$ [8].

The effect of a lattice on $\lambda_{0, j}^{(V)}$ was studied in e.g., [4, 5, 6, 7, 9], where it was shown that if the soliton is centered at a lattice minimum (maximum), then $\lambda_{0, j}^{(V)}$ becomes positive (negative), hence the spectral condition is satisfied (violated).

In [4, 5, 6, 7] it was observed numerically that violation of the spectral condition results in a drift instability, i.e., the center of mass (COM) of the beam in the $x_{j}$ coordinate, defined as $\overline{x_{j}}(z)=\int x_{j}|A|^{2} / \mathcal{P}$, drifts away from its initial location $\overline{x_{j}}(0)$ near the lattice maximum. So far, however, the relation between the spectral condition and the drift instability has not been established analytically. To do that, we note that since $f_{j}^{(V)}$ and $g_{j}^{(V)}$ are odd [4],

$$
\begin{aligned}
\overline{x_{j}}(z) & =\frac{1}{\mathcal{P}}\left\langle x_{j},|u(\mathbf{x} ; \mu)+h(z, \mathbf{x})|^{2}\right\rangle \\
& =B\left(c_{1} e^{\Omega_{j} z}+c_{-1} e^{-\Omega_{j} z}\right),
\end{aligned}
$$

where $B=2\left\langle x_{j}, u f_{j}^{(V)}\right\rangle / \mathcal{P}$ is constant. Thus,

$$
\ddot{x_{j}}(z)=\Omega_{j}^{2} \overline{x_{j}}(z) .
$$

Relation (5) shows that a failure to satisfy the spectral condition $\left(\lambda_{0, j}^{(V)}<0\right)$ leads to a drift instability. Moreover, the magnitude of $\Omega_{j}=\left|C_{V} \lambda_{0, j}^{(V)}\right|^{\frac{1}{2}}$ determines the drift rate away from the lattice maximum in the $x_{j}$ direction.

A simpler expression for $C_{V}$ can be obtained for a weak lattice $(V \ll \mu)$ and a power nonlinearity $F=|A|^{p-1}$. In this case, $f_{j}^{(V)} \cong f_{j}^{(V \equiv 0)}=\frac{\partial Q}{\partial x_{j}}$ and $L_{-, \mu}^{(V)} \cong L_{-, \mu}^{(V \equiv 0)}=$ $\mu L_{-, 1}^{(V \equiv 0)}$. Thus, $C_{V} \cong \mu\left\langle\frac{\partial Q}{\partial x_{j}}, \frac{\partial Q}{\partial x_{j}}\right\rangle /\left.\left\langle L_{-, \mu}^{-1} \frac{\partial Q}{\partial x_{j}}, \frac{\partial Q}{\partial x_{j}}\right\rangle\right|_{\mu=1}$. From the Pohozaev identities it follows that $\left\langle\frac{\partial Q}{\partial x_{j}}, \frac{\partial Q}{\partial x_{j}}\right\rangle=$ $\frac{1}{4 \delta}\langle Q, Q\rangle$ where $\delta=\frac{(2-d) p+2+d}{4(p-1)}$. In addition, if we multiply $L_{-, \mu} w=\frac{\partial Q}{\partial x_{j}}$ by $x_{j} Q$ and integrate in parts we get $\left\langle L_{-, \mu}^{-1} \frac{\partial Q}{\partial x_{j}}, \frac{\partial Q}{\partial x_{j}}\right\rangle=\frac{1}{4}\langle Q, Q\rangle$. Substituting in (3) gives

$$
\Omega_{j}^{2} \cong-\frac{\mu}{\delta} \lambda_{0, j}^{(V)}
$$

Hence, for a weak lattice, the dependence of the drift rate $\Omega_{j}$ on the lattice period $N$ is only through its effect on $\lambda_{0}^{(V)}$. The approximation (6) can be generalized for different nonlinearities and to lattices which are not weak. For example, in the case of narrow lattice, $\Omega_{j}^{2}$ is given by (6) with $\mu$ replaced by $\mu+V\left(\mathbf{x}_{0}\right)$ where $\mathbf{x}_{0}$ is the location of the soliton peak [5].

We solve Eq. (11) numerically for $F=|A|^{2}, d=1$ and

$$
V(x)=V_{0} \cos (2 \pi N x),
$$

with the initial condition $A(0, x)=u(x-\delta)$, where $u(x)$ is the solution of (2) centered at $x=0$. Therefore, $\bar{x}(z=$ $0)=\delta($ since $d=1$, we can suppress the index $j)$. For a small shift $\delta \ll 1$, we can rewrite $A(0, x)=u(x)+h(0, x)$ where $h(0, x)=-\delta \frac{d u}{d x}+\mathcal{O}\left(\delta^{2}\right)$. Since $h(0, x)=\left(c_{1}+\right.$ $\left.c_{-1}\right) f^{(V)}+i\left(c_{1}-c_{-1}\right) g^{(V)}$, then $c_{1}-c_{-1}=0$ and by (4),

$$
\bar{x}(z)=\delta \cosh \Omega z .
$$

In our simulations we observe that that the COM evolves according to (8), see Fig. 1(a), and therefore, calculate the drift rate numerically by finding the best fitting $\Omega$. We fix $V_{0}=0.1$ and $\mu=4.5$ and vary $N$. As expected for a soliton centered at a lattice maximum [4, 5], $\lambda_{0}^{(V)}<0$ for all values of $N$, and $\lambda_{0}^{(V)}$ vanishes in the limits $N \rightarrow 0$ (narrow solitons) and $N \rightarrow \infty$ (wide solitons), see Fig. 1(b). In Fig. 1(c) we confirm that the numerically computed drift rate $\Omega$ is in excellent agreement with Eq. (3) and also with the approximation (6). Accordingly, each value of $\lambda_{0}^{(V)}$ is attained at two different values of $N$ for which the drift rates are nearly identical. Indeed, in Fig. 1(a) we see that the drift rate of the COM when $N=0.2$ and $N=1.437$, both for which $\lambda_{0}^{(V)} \cong-0.0454$, is the same over more than 3 orders of magnitude and 40 diffraction lengths. In Fig. 1(d) we repeat these simulations with a stronger lattice $\left(V_{0}=2\right)$. In this case, the numerically computed drift rate is in excellent agreement with the one predicted by Eq. (3). The approximation (6) is very accurate only for narrow $(N \ll 1)$ and wide $(N \gg 1)$ solitons. Indeed, although the lattice oscillations are not small, for narrow and wide solitons, the effect of a mean-zero lattice is weak, hence the deviation of $f_{j}^{(V)}$ from $\frac{\partial Q}{\partial x_{j}}$ is small [4, 5]. Although for solitons of $N=\mathcal{O}(1)$ width the deviation of $f_{j}^{(V)}$ from $\frac{\partial Q}{\partial x}$ is not small, the approximation (6) is, at most, $10 \%$ inaccurate.

In Fig. 11(e) we fix $N=1$ and $V_{0}=0.1$ and vary $\mu$. As in Fig. 1(b), since the soliton is centered at a lattice maximum, $\lambda_{0}^{(V)}<0$ for all values of $\mu$, and $\lambda_{0}^{(V)}$ vanishes in the two limits $\mu \rightarrow 0$ (wide solitons) and $\mu \rightarrow \infty$ (narrow solitons). In Fig. 1(f) we see that $\Omega$ is monotonically increasing in $\mu$, and that the numerically calculated drift rate is in excellent agreement with the analytical prediction (3) and also with its approximation (6). 
We emphasize that despite the similarity of the dependence of $\lambda_{0}^{(V)}$ on $N$ and $\mu$ (see Fig. 1(b) and Fig. 1(e)), the dependence of $\Omega$ on $N$ and $\mu$ is completely different in the narrow-beam limit. Indeed, for narrow beams $\left.\lambda_{0}^{(V)} \cong 4 \delta \frac{N^{2}}{\mu} \frac{d^{2} V}{d x^{2}}\right|_{x=0}$ [5] so that by ([6), $\Omega^{2} \approx \Omega_{\text {narrow }}^{2}=$ $-\left.4 N^{2} \frac{d^{2} V}{d x^{2}}\right|_{x=0}$. Hence, $\Omega$ vanishes for a fixed $\mu$ and $N \rightarrow 0$ (Fig. 1(c)) but approaches $\Omega_{\text {narrow }} \cong 2.8$ for a fixed $N$ and $\mu \rightarrow \infty$ [Fig. [1(f)].

In order to show that our results are also valid in higher dimensions, we solve Eq. (11) in a $d=2$ setting with

$$
V(x, y)=\frac{V_{0}}{2}\left(\cos ^{2}(2 \pi x)+\cos ^{2}(2 \pi y)\right),
$$

with $V_{0}=5$, and find the numerical drift rate to be in excellent agreement with Eq. (3), see Fig. 1(g). Remarkably, although the lattice is strong, the numerical drift rate is also in excellent agreement with the approximation (6) in which $\mu$ is shifted by $V_{0} / 2$, the mean of $V$.
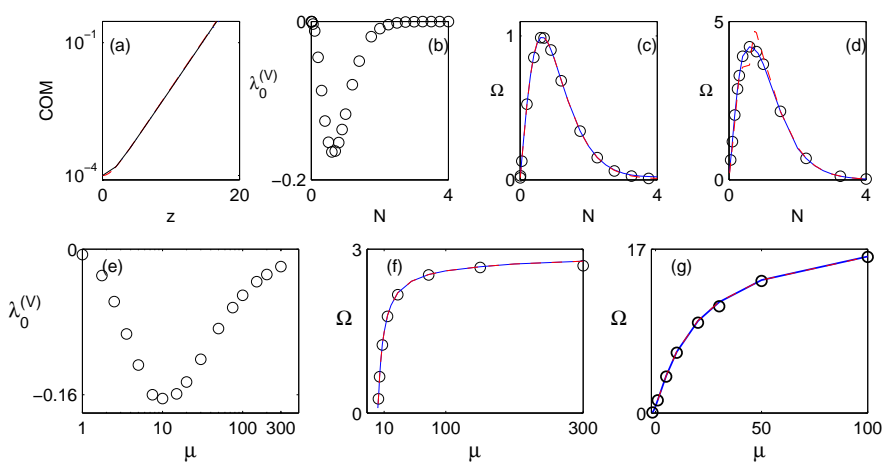

FIG. 1: (Color online) (a) The dynamics of the COM for $V_{0}=0.1, \delta=10^{-4}$ and the lattice (7) for $N=0.2$ (dashed red line), $N=1.437$ (dotted blue line), and the analytical prediction (8) with $\Omega \sim 0.52$ (black solid line). The 3 lines are indistinguishable. (b) $\lambda_{0}^{(V)}$ as a function of $N$. (c) Drift rate $\Omega$ as a function of $N$. The analytical prediction (3) (solid blue line) and its approximation (6) (dashed red line) are nearly indistinguishable. (d) Same as (c) for $V_{0}=2$. (e) $\lambda_{0}^{(V)}$ as a function of $\mu$. (f) Same as (c), but as a function of $\mu$. (g) Same as (f) for $d=2$ and the lattice (9) with $V_{0}=5$.

The analytical relation (8), together with (3) or (6), enable us to estimate the distance at which a soliton initially centered near a lattice maximum will deviate significantly from its initial location. In particular, if the initial shift $\delta$ and/or drift rate $\Omega$ are sufficiently small, then this "mathematically unstable" soliton can remain "near" its initial location over the propagation distance of the experiment, i.e., be "physically stable". This observation can explain the experimental results of [3], where solitons centered at a lattice maximum did not drift over $\approx 18$ diffraction lengths.

The relation between the sign and magnitude of the perturbed near-zero eigenvalues $\left\{\lambda_{0, j}^{(V)}\right\}_{j=1}^{d}$ and the drift instability appears to be universal. Indeed, we now show that it also occurs in numerical calculation of soliton profiles using Petviashvili's iterations method (PIM), which is nowadays frequently used in optics and BEC [10]. In [11] it was proved that PIM converges only if $L_{+, \mu}^{(V)}$ has at most one negative eigenvalue, which is a spectral condition similar to the one for the stability of NLS solitons. Accordingly, PIM is not expected to converge for solitons centered at lattice maxima.

We solve Eq. (2) with $F=|u|^{2}$ using PIM with the initial guess $u^{(0)}=u(x-\delta)$, where $u(x)$ is the solution of (2) centered at a maximum of the lattice (7) with $V_{0}=0.1$. Similarly to the dynamics of NLS solitons centered slightly off a lattice maximum, the COM of $u^{(m)}$ evolves according to $\bar{x}(m) \sim \delta e^{\Omega m}$ (data not shown), where $u^{(m)}$ is the solution in the $m$ th iteration. Thus, we conclude that when the spectral condition for PIM is violated, the method does not converge because the iterative solution drifts away from the lattice maximum. In that sense, the analogy between the dynamics (in $z$ ) of NLS solitons and of $u^{(m)}$ (in $m$ ) is further demonstrated, since in both cases, violation of the spectral condition leads to a drift instability. We also compute the exponential drift rate $\Omega$ numerically for various combinations of $N$ and $\mu$ and observe that $\Omega^{2} \cong-D_{V}\left(\lambda_{0}^{(V)}(N, \mu) / \mu\right)^{2}$ where the constant $D_{V}$ depends on $V_{0}$ but is independent of $\mu$ and $N$, see Fig. 2(a). Interestingly, the scaling of $\Omega$ in $\lambda_{0}^{(V)}$ and in $\mu$ is different from ([6), yet in both cases $\Omega$ depends on $N$ only through $\lambda_{0}^{(V)}$.

Although in [11] it was proved that the iterations should diverge for solitons centered at a lattice maximum, in several studies these iterations did "converge" [4, [5, 12]. To explain this apparent inconsistency, in Fig. 2(b) we plot $\max _{x}\left|u^{(m)}-u\right|$ as a function of $m$ for $N=0.2, \mu=2$ and the lattice (7) with $V_{0}=0.1$ and $u^{(0)}=e^{-x^{2}}$, and observe that the iterations converge (i.e., $\max _{x}\left|u^{(m)}-u\right|<10^{-13}$ ) after $\approx 40$ iterations. However, if we continue the iterations, a significant drift of the COM occurs around $m \approx 2000$. To understand this "post-convergence" drift, we note that in this example, $\lambda_{0}^{(V)} \cong-0.085$ and $\Omega \cong 0.018$. Since the seed of the drift is roundoff error, then $\bar{x}(m=0)=\mathcal{O}\left(10^{-16}\right)$. Indeed, $10^{-16} e^{0.018 \cdot 2000}=\mathcal{O}(1)$. This example of a numerical iterative solution which theoretically should diverge yet in practice converges is thus analogous to the mathematically unstable yet physically stable NLS solitons discussed earlier.
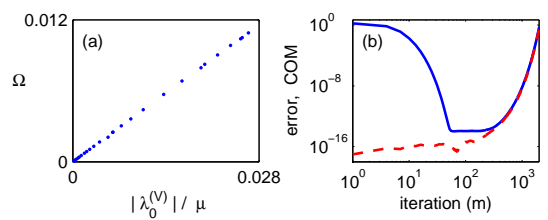

FIG. 2: (Color online) (a) Drift rate $\Omega$ as a function of $\lambda_{0}^{(V)} / \mu$ for the solution of (2) using PIM. (b) Maximal error (solid line) and COM (dashed red line) in the $m$ th iteration.

We now consider solitons centered near a lattice min- 
imum. Since $\lambda_{0, j}^{(V)}>0$, the spectral condition is satisfied. Hence, these solitons are stable under small lateral perturbations. Indeed, relation (5) shows that small lateral perturbations would lead to oscillations around the lattice maximum, while relation (3) shows that the magnitude of $\lambda_{0, j}^{(V)}$ determines the strength of the restoring force. For example, consider a soliton centered at a lattice minimum which is launched at an angle $\theta_{j}$ between the $x_{j}$ and $z$ axes. Such an angle corresponds to an initial transverse velocity of $v_{0, j}=\dot{\overline{x_{j}}}(z=0)=\tan \theta_{j}$. By (3)-(4), the COM evolves according to

$$
\overline{x_{j}}(z)=v_{0, j} \sin \left(\left|\Omega_{j}\right| z\right) /\left|\Omega_{j}\right| .
$$

Thus, as $\lambda_{0, j}^{(V)}$, hence $\left|\Omega_{j}\right|$, increase, the maximal deviation of the COM from the lattice minimum becomes smaller, implying stronger lateral stability.

Eq. (10) gives an accurate description of the dynamics for small velocities. However, for non-small velocities, as the soliton propagates sideways, the attraction towards the lattice minimum decreases, an effect which is not captured by Eq. (10). To see that, we solve Eq. (1) with $d=2, F=|A|^{2}$ and the lattice (9) with $V_{0}=0.5$. The initial condition is $A(0, x, y)=u(x, y) e^{i\left(v_{0} x+v_{0} y\right) / 2}$, i.e., a soliton centered at a lattice minimum $\mathbf{x}_{\min }=(0,0)$ with initial velocity in the direction of the nearest lattice maximum at $\mathbf{x}_{\max }=(0.25,0.25)$. Indeed, for small initial velocities, the agreement between the dynamics and Eq. (10) is excellent, see Fig. 3(a). For higher velocities, the COM initially evolves according to Eq. (10) but deviates from it as it approaches the lattice maximum, see Fig. 3(b). For a sufficiently large initial velocity, the soliton can "tunnel" beyond the nearest lattice maximum. The critical velocity for tunneling $\mathbf{v}_{0}^{c r}$ is the one for which the transverse velocity $\dot{\overline{\mathbf{x}}}(z)$ vanishes at the lattice maximum. The upper limit $\left|\mathbf{v}_{0}^{c r}\right| \leq$ $v_{t h}^{c r}=\sqrt{\sum_{j=1}^{d}\left|\Omega_{j}\right|^{2}\left(\mathbf{x}_{\max }-\mathbf{x}_{\min }\right)_{j}^{2}}$ can be derived from Eq. (10). In the case of Fig. [3(b), this bound gives $\left|\mathbf{v}_{0}^{c r}\right| \leq$ 1.22 , an over-estimate of $\approx 45 \%$ over $\left|\mathbf{v}_{0}^{c r}\right| \approx 0.485 \sqrt{2}$.
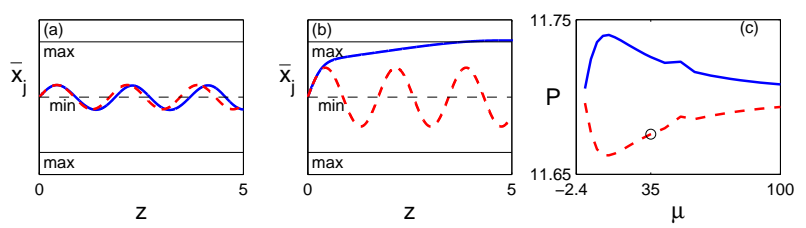

FIG. 3: (Color online) Dynamics of $\bar{x}_{1}\left(=\bar{x}_{2}\right)$ (solid) and theoretical prediction (10) (dashes) in a bulk medium with $F=|A|^{2}$, the lattice (9) with $V_{0}=0.5, \mu=35$ and (a) $\mathbf{v}_{0}=(0.2,0.2),(b) \mathbf{v}_{0}=(0.485,0.485)$. (c) Power of solitons centered at a lattice maximum (solid) and minimum (dashes).

The standard formula for $\mathbf{v}_{0}^{c r}$, based on the PeierlsNabarro potential (PNP) approach 2], is $\left|\mathbf{v}_{0}^{c r}\right|=$ $\sqrt{4 \Delta \mathcal{H} / \mathcal{P}}$ where $\Delta \mathcal{H}$ is the difference in the Hamiltonians of equal-power solitons centered at a lattice minimum
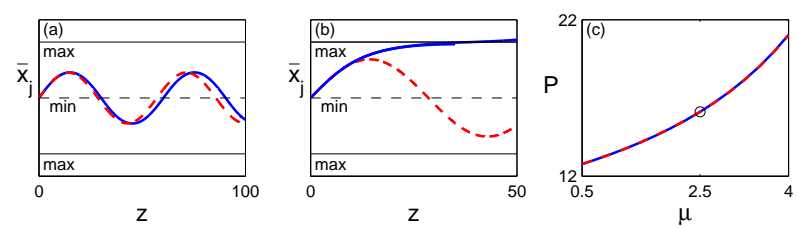

FIG. 4: (Color online) Same as Fig. 3 for $F=|A|^{2}-0.02|A|^{4}$, $\mu=2.5, V_{0}=1$ and (a) $\mathbf{v}_{0}=(0.0125,0.0125)$, (b) $\mathbf{v}_{0}=$ $(0.01905,0.01905)$.

and maximum, respectively. In order to apply the PNP approach, the power of the soliton centered at a lattice maximum should be equal to that of a soliton centered at a lattice minimum. For a two-dimensional Kerr medium $\left(F=|A|^{2}\right)$, however, such "soliton pairs" do not exist, since the power of all solitons centered at a lattice maximum is below that of all solitons centered at a lattice minimum, see Fig. 3(c). Therefore, one cannot use the PNP approach, and the upper bound $v_{t h}^{c r}$ provides the only analytic estimate of $\left|\mathbf{v}_{0}^{c r}\right|$.

Finally, we solve Eq. (11) for a cubic-quintic nonlinearity and the lattice (9). As in the Kerr case, for small initial velocities, the agreement between the numerics and Eq. (10) is excellent over many diffraction lengths [Fig. 4(a)], while for higher velocities the COM deviates from Eq. (10) as the soliton approaches the lattice maximum [Fig. 4(b)]. In this case the PNP approach is applicable [see Fig. 4(c)] and yields $\left|\mathbf{v}_{0}^{c r}\right| \simeq 0.027$. The value of the critical velocity obtained numerically is within $1 \%$ of the PNP prediction [see Fig. 4(b)]. To the best of our knowledge, this is the first demonstration of quantitative agreement of the PNP approach with numerical results for $d=2$. The research of G.F. and Y.S. was partially supported by BSF grant no. 2006-262.

[1] A.B. Aceves et al., Phys. Rev. E 53, 1172 (1996). Y. Kartashov et al., Opt. Lett. 29, 766 (2004). Z. Xu et al., Phys. Rev. Lett. 95, 113901 (2005). R. Vicencio and M. Johansson, Phys. Rev. E 73, 046602 (2006). G. Dekel et al., Phys. Rev. A 75, 043617 (2007).

[2] Y. Kishar and D. Campbell, Phys. Rev. E 48, 3077 (1993).

[3] R. Morandotti et al., Phys. Rev. Lett. 83, 2726 (1999).

[4] G. Fibich and Y. Sivan and M. Weinstein, Physica D 217, 31 (2006). Y. Sivan and G. Fibich and M. Weinstein, Phys. Rev. Lett. 97, 193902 (2006).

[5] Y. Sivan et al., http://arxiv.org/abs/0707.1589.

[6] S. Le-Coz et al., http://arxiv.org/abs/0707.2491

[7] D. E. Pelinovsky et al., Phys. Rev. E. 70, 036618 (2004).

[8] M. Weinstein, SIAM J. Math. Anal. 16, 472 (1985). M. Grillakis, Comm. Pure Appl. Math. 41, 747 (1988).

[9] Z. Rapti et al., J. Phys. A. 40, 14151 (2007).

[10] V. Petviashvili, Sov. J. Plasma Phys. 2, 257 (1976). M. Ablowitz and Z. Musslimani, Opt. Lett. 30, 2140 (2005).

[11] D. E. Pelinovsky and Y. A. Stepanyants, SIAM J. Nu- 
mer. Anal. 42, 1110 (2004). T.I. Lakoba and J. Yang, J. Comput. Phys., 226, 1668 (2007).

[12] M. Ablowitz et al., Phys. Rev. E 74, 035601(R) (2006). 